\title{
Isolation and Thermal Characterization of Softwood- Derived Lignin with Thermal Flow properties
}

Thi Thi Nge, Eri Takata, Shiho Takahashi, and Tatsuhiko Yamada*

Forestry and Forest Products Research Institute, 1 Matsunosato, Tsukuba, Ibaraki 305-8687, Japan

*Corresponding author: yamadat@affrc.go.jp

\section{Contents}

List of Scheme and Figures

Scheme S1. Schematic illustration of acid-catalyzed PEG solvolysis process

Figure S1. TGA profiles of glycol lignin (A and B) at varying acid (SA) catalyst concentrations for PEG200 solvolysis process, and (C and D) at varying PEG polymer chain lengths with SA $0.5 \%$ at $150^{\circ} \mathrm{C}$ for $2 \mathrm{~h} .(\mathrm{A}, \mathrm{C}=$ weight loss curves and $\mathrm{B}, \mathrm{D}=$ derivative weight loss, $\mathrm{DTG}$, curves)

Figure S2. TGA profiles of PEG200 and PEG600 using as solvolysis reagent 


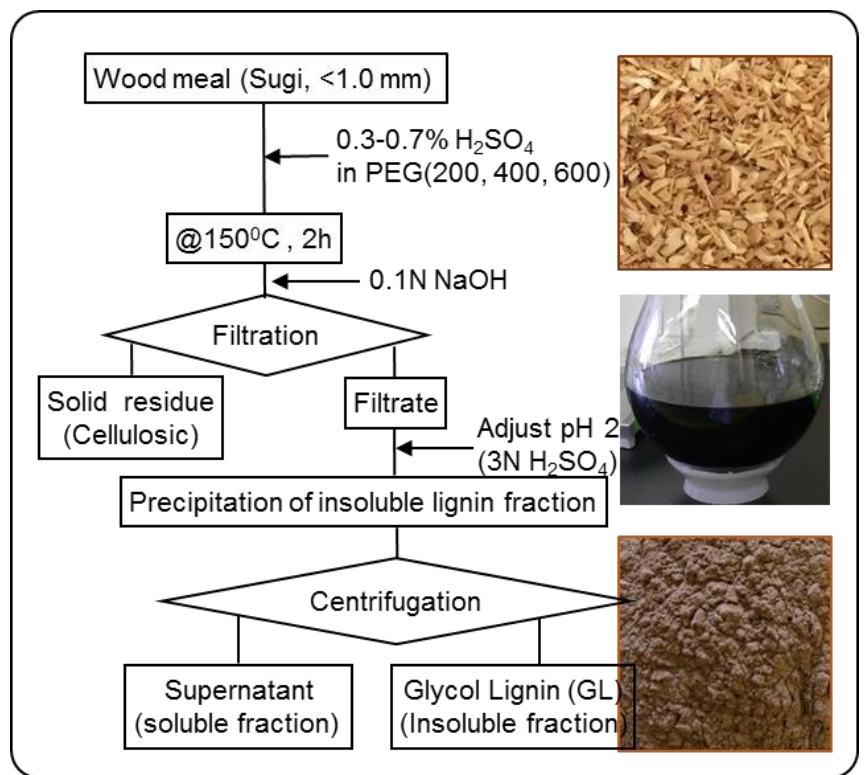

Scheme S1. Schematic illustration of acid-catalyzed PEG solvolysis process

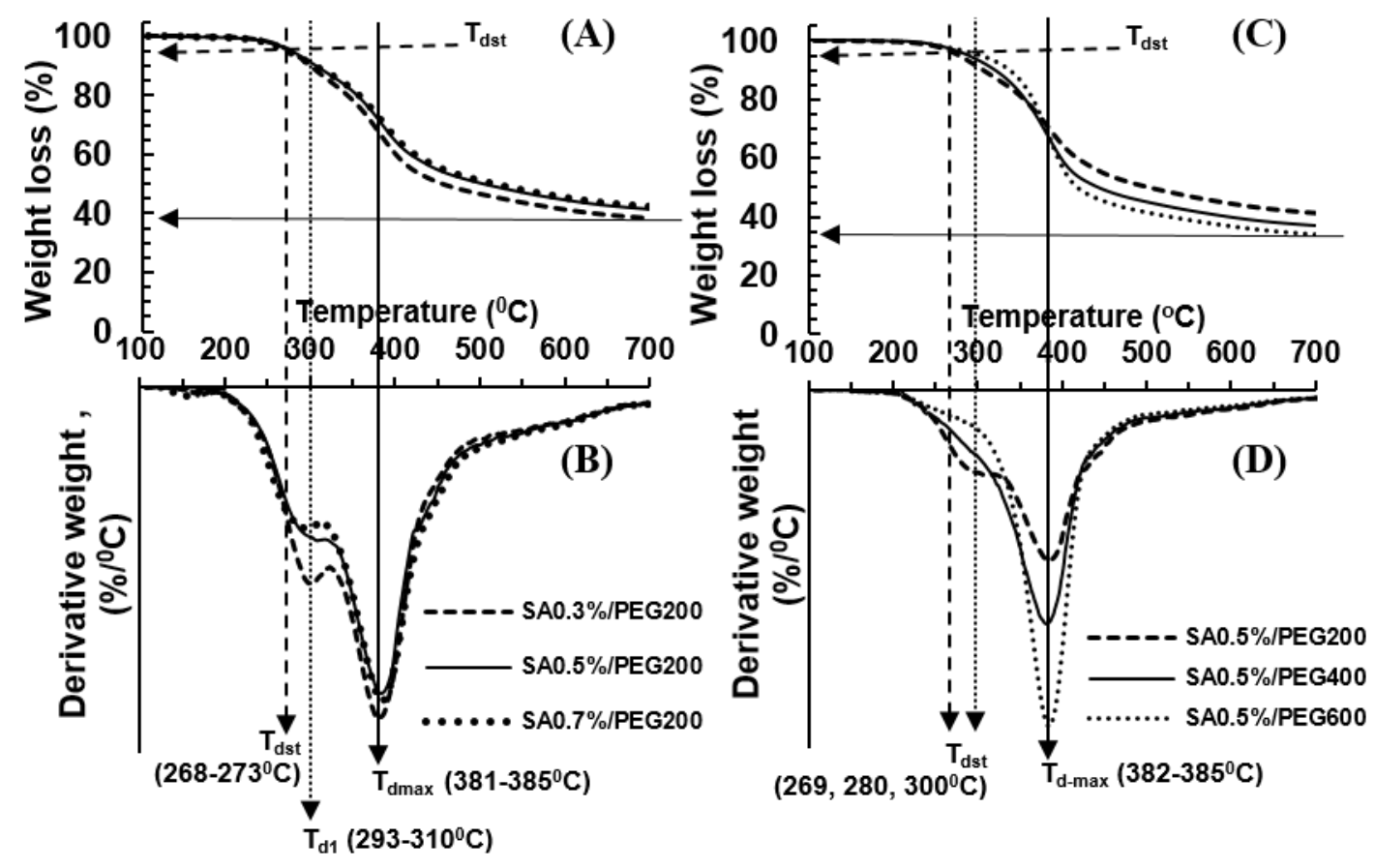

Figure S1. TGA profiles of glycol lignin (A and B) at varying acid (SA) catalyst concentrations for PEG200 solvolysis process, and (C and D) at varying PEG polymer chain lengths with SA $0.5 \%$ at $150^{\circ} \mathrm{C}$ for $2 \mathrm{~h}$. ( $\mathrm{A}, \mathrm{C}=$ weight loss curves and $\mathrm{B}, \mathrm{D}=$ derivative weight loss, $\mathrm{DTG}$, curves) 


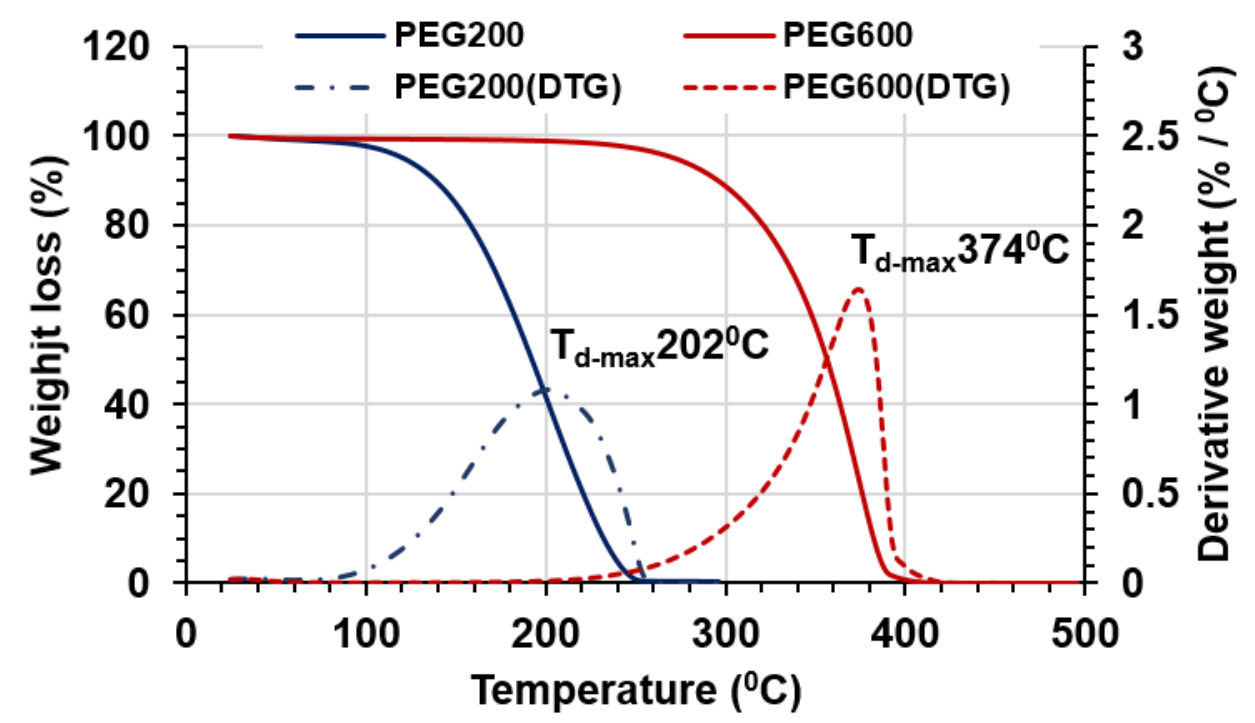

Figure S2. TGA profiles of PEG200 and PEG600 using as solvolysis reagent 